\title{
Effects of Hand Holding on Anxiety and Pain During Prostate Biopsies: A Pilot Randomized Controlled Trial
}

\section{Wenfeng Li* \\ Yuanshen Mao* \\ Yufei Gu \\ Chao Lu \\ Xin Gu \\ Bao Hua \\ Weixin Pan \\ Qinghong $X i$ \\ Bin Xu}

Department of Urology, The Ninth People's Hospital Affiliated to Shanghai Jiao Tong University, Shanghai, People's Republic of China

*These authors contributed equally to this work
Correspondence: Bin Xu; Qinghong Xi Department of Urology, The Ninth People's Hospital Affiliated to Shanghai Jiao Tong University, Shanghai, People's Republic of China

Tel +862I56691101

Email kuangfeng06I2@I26.com;

|361|829161@I63.com
Background: Effective pain management is limited for patients during prostate biopsy (PBx). Touch support, such as hand holding, has stress-buffering benefits and effective analgesic effects. We conducted a prospective, single-center randomized clinical trial to assess whether hand holding can reduce patient anxiety, pain, and dissatisfaction during PBx. Methods: Between April 2020 and October 2020, 120 male patients were randomized into three groups: a hand holding with relatives (HR) group, a hand holding with strangers (HS) group and a control group. A visual analog scale (VAS) was used for self-assessments of pain and satisfaction. Anxiety levels were quantified according to the State-Trait Anxiety Inventory (STAI). Hemodynamic changes were also measured.

Results: The degree of pain and anxiety in the hand-holding groups was significantly better than that in the control group $(P<0.001)$, and the patients were more willing to undergo repeat $\mathrm{PBx}(P=0.017)$. The anxiety levels in the HR group were significantly lower than those in the HS group $(P=0.019)$. During $\mathrm{PBx}$, the changes in systolic blood pressure and heart rate in the hand-holding groups were more stable than those in the control group $(P<0.01)$, and the fluctuations in heart rate in the HR group were smaller than those in the HS group $(P<0.01)$.

Conclusion: Hand holding, especially with relatives, can promote incremental reductions in anxiety, pain and dissatisfaction in patients during PBx. Hence, we recommend hand holding with relatives as an effective adjunct during PBx.

Keywords: anxiety, transrectal prostate biopsy, hand holding, pain, satisfaction

\section{Introduction}

The introduction of transrectal ultrasound (TRUS)-guided prostate biopsy (PBx) was a major advancement in diagnostic methods for detecting prostate cancer (PCa). However, severe pain causes patients to move during the procedure, which increases the incidence of complications and interrupts the operation. ${ }^{1}$ Twenty percent of men who were about to undergo PBx experienced great stress and/or anxiety, and 18\% would not undergo a repeat biopsy associated with significant pain or discomfort. ${ }^{2,3}$ Due to the need for repeated biopsy and the large number of samples needed, effective pain management for TRUS-PBx is paramount, but the best anesthesia method is not yet clear. ${ }^{4,5}$

Recently, the effectiveness of music therapy has been evaluated for biopsies and other painful procedures and has been shown to usefully reduce anxiety and pain. ${ }^{6}$ Social support, such as hand holding, also has stress-buffering benefits and effective 
analgesic effects on pain. ${ }^{7,8}$ Holding hands with a spouse further reduces the threat response. ${ }^{9}$

Some studies have shown that having nurses hold the patient's hand during percutaneous vertebroplasty and cardiac intervention can relieve anxiety and pain under local anesthesia. ${ }^{10}$ There are no controlled randomized clinical trials that have determined the efficacy of hand holding in patients with TRUS-PBx. Therefore, we designed a randomized clinical trial to determine whether a handholding intervention would be effective in reducing patient anxiety for TRUS-PBx.

\section{Patients and Methods Design and Setting}

We designed a nonblinded, single-center randomized clinical trial. The study was carried out in the Department of Urology of the Ninth People's Hospital Affiliated with Shanghai Jiao Tong University in Shanghai, China, from April to October 2020. This study was performed in line with the principles of the Declaration of Helsinki. Approval was granted by the Ethics Committee of the Ninth People's Hospital, Shanghai Jiao Tong University School of Medicine (No. SH9H-2020-T40-1) and registered with clinicaltrials.gov (ChiCTR2000032238).

\section{Eligibility Criteria}

Participants who met one of the following inclusion criteria were eligible for the study: (1) prostate-specific antigen $(\mathrm{PSA})>10.0 \mathrm{ng} / \mathrm{mL}$; (2) PSA $>4.0 \mathrm{ng} / \mathrm{mL}$ and free/ total PSA ratio $<0.2$; (3) PSA velocity $>0.75 \mathrm{ng} / \mathrm{mL} /$ year; and (4) an abnormal digital rectal examination (DRE) or suspicious areas found with imaging (TRUS, MRI). The exclusion criteria consisted of several parameters: (1) diseases prone to severe bleeding; (2) acute infection and fever; (3) severe anorectal disease (fistula, hemorrhoids, etc.); (4) hypertensive crisis; (5) decompensation period of cardiac dysfunction; (6) unstable blood glucose stage of diabetes; (7) use of analgesics or sedative drugs within 24 hours before TRUS-PBx; (8) allergy to oxybuprocaine; and (9) significant communication difficulties, severe hearing loss, or inability to complete the required surveys.

\section{Procedure}

All patients in the study underwent the standard TRUS-PBx procedure at the Urology Department of our hospital. The patient was placed in a right lateral decubitus position. Ten milliliters of $3 \%$ oxybuprocaine gel was introduced into the rectum 8 min before insertion of the TRUS probe according to the drug instructions. Rectal prostate biopsy probes (Mindray 6CV1s, Shenzhen, China) and coaxial puncture biopsy needles (Achieve A1825, Merit Medical, South Jordan, Utah, USA) were used, and a 12-core biopsy was performed. All TRUS-PBx procedures were performed by a single well-experienced urologist. During the procedures, patients did not take any painkillers or sedatives.

The patients who met the inclusion criteria were assigned to one of the three groups: (1) the hand holding with relatives (HR) group, (2) the hand holding with strangers (HS) group, or (3) the control group. Participants in the HR group had a relative (spouse or child) hold one of their hands during the TRUS-PBx. Participants randomized to the HS group were touched by a nurse until the TRUS-PBx was complete. Participants in the control group had treatment as usual and did not have their hands held. The urologist only touched the participant to the extent necessary to perform the procedure.

\section{Sample Size}

The sample size was determined to be at least 111 participants with 5\% alpha and $20 \%$ beta errors (http://power andsamplesize.com). The criteria used for the sample size calculation were mean visual analog scale (VAS) scores of 4.66 and 6.29 with standard deviations (SDs) of 2.03 and 2.49 for the intervention group and control group, respectively. To account for possible dropout, a total of 126 patients were planned.

\section{Randomization and Allocation}

Participants were randomized 1:1:1 to 1 of the 3 groups, with 42 patients in each group, by the block randomization method. Randomization was performed according to a computer-generated randomized grouping list (https:// www.sealedenvelope.com) by the advisory biostatistician at our hospital.

\section{Outcomes}

The study was divided into three stages: before, during and after TRUS-PBx. The stages were $15 \mathrm{~min}$ before the operation of TRUS-PBx, during the TRUS-PBx process, and 15 min after the completion of TRUS-PBx.

Before and after the TRUS-PBx, the nurse assisted the participants in using the VAS to measure pain intensity and satisfaction. The State-Trait Anxiety Inventory (STAI) was used to measure anxiety levels before and after TRUSPBx. The patient's hemodynamic parameters, including 
systolic and diastolic blood pressure (before and after TRUS-PBx) and heart rate (before, during and after TRUS-PBx), were measured.

The primary outcome was the differences in pain intensity based on the VAS and anxiety levels based on the STAI compared with baseline for each group. The VAS scores ranged from 0 to 10: 10 indicated extreme pain, and 0 indicated no symptoms. The 6-item STAI is a measure of anxiety that has been used to assess participants' anxiety in a fast-paced clinical setting. ${ }^{11}$ It was derived from the 20-item STAI, which assesses state anxiety associated with the present situation and trait anxiety related to an individual's personality. Scores ranged from 6 (least upset) to 24 (most upset). Participants completed the VAS and STAI before and immediately after the procedure.

The secondary outcomes were the differences in satisfaction, based on the VAS score reported by the participants at the end of TRUS-PBx, and hemodynamics for each group. Participants' satisfaction was measured on a Likert scale of 1 to 10 , ranging from very dissatisfied to very satisfied. Hemodynamic parameters included systolic blood pressure (SBP), diastolic blood pressure (DBP), and heart rate.

\section{Statistical Analysis}

Statistical analysis was performed using SPSS 25.0 software (IBM Corp., Armonk, NY, USA). The KolmogorovSmirnov test was used to distinguish whether data were normally distributed. One-way ANOVA or Kruskal-Wallis test was selected to compare the differences among three groups according to the normality of the data. If the difference was statistically significant, the least significant difference $t$-test or Mann-Whitney $U$-test was used to further compare the differences between the two groups (HR vs HS). A value of $5 \%$ was considered the threshold for significance.

\section{Results}

A total of 137 patients were assessed for eligibility, and none of them suffered from neurological diseases such as paraplegia and hemiplegia. Eleven participants declined to participate, and 6 participants did not complete the survey correctly. A total of 120 patients were prospectively randomized into the HR group $(n=40)$, the HS group $(n=39)$ and the control group $(n=41)$. Patient enrollment, randomization, and analyses are presented in a Consolidated Standards of Reporting Trials flow diagram.

There was no significant difference in mean age, weight, marriage status, education or employment status. The three groups were well matched, and no significant intergroup differences were found for patient clinicopathologic characteristics, which included prostate volume, PSA level, International Prostate Symptom Score and Quality of Life score (Table 1). The reasons for TRUS-PBx in the three groups were similar $(P=0.834)$; the most common reason was elevated PSA, followed by abnormal imaging and abnormal DRE. Similarly, there was no significant difference in the average time of TRUS-PBx among the three groups $(P=0.361)$.

The comparison of the VAS pain and STAI anxiety scores among the three groups of participants in the study is shown in Table 2. Pain intensity, measured by VAS during TRUS-PBx, was significantly lower in the hand-holding groups $(P<0.001)$ than in the control group.

Table I Comparison of Clinicopathologic Characteristics $(n=\mid 20)$

\begin{tabular}{|c|c|c|c|c|c|}
\hline & HR Group $(n=40)$ & HS Group (n=39) & Control Group $(n=4 I)$ & Statistics & $P$-value \\
\hline Prostate volume, $\mathrm{mL}$, mean $\pm \mathrm{SD}$ & $57.2 \pm 19.6$ & $53.2 \pm 17.8$ & $55.2 \pm 17.7$ & 0.471 & 0.626 \\
\hline PSA level, ng/mL, median (IQR) & $12.1(11.1)$ & $10.0(15.0)$ & $11.3(10.0)$ & 1.680 & 0.432 \\
\hline IPSS score, mean \pm SD & $22.6 \pm 5.4$ & $22.0 \pm 5.2$ & $22.9 \pm 3.8$ & 0.382 & 0.683 \\
\hline QOL score, mean \pm SD & $4.1 \pm 0.6$ & $4.3 \pm 0.7$ & $4.2 \pm 0.8$ & 0.837 & 0.436 \\
\hline Previous biopsies, $n(\%)$ & $7(17.5)$ & $12(30.8)$ & $9(22.0)$ & 2.010 & 0.366 \\
\hline \multicolumn{6}{|l|}{ Reasons for biopsy, $n$} \\
\hline Elevated PSA & 37 & 32 & 40 & 1.460 & 0.834 \\
\hline Abnormal findings on DRE & 7 & 10 & 7 & & \\
\hline Abnormal imaging & 20 & 18 & 22 & & \\
\hline Procedure time, min, mean \pm SD & $11.8 \pm 2.7$ & $I I . I \pm 2.4$ & $11.6 \pm 2.6$ & 1.027 & $0.36 \mathrm{I}$ \\
\hline
\end{tabular}

Abbreviations: HR, hand holding with relatives; HS, hand holding with strangers; SD, standard deviation; PSA, prostate-specific antigen; IQR, interquartile range; IPSS, international prostate symptom score; QOL, quality of life; DRE, digital rectal examination. 
Table 2 Comparison of Periprocedural Outcomes (Anxiety and Pain) Among Three Groups ( $n=120)$

\begin{tabular}{|c|c|c|c|c|c|c|c|}
\hline & $\begin{array}{l}\text { HR Group } \\
(n=40)\end{array}$ & $\begin{array}{l}\text { HS Group } \\
(n=39)\end{array}$ & $\begin{array}{l}\text { Control } \\
\text { Group }(n=4 I)\end{array}$ & $\begin{array}{l}\text { Statistics } \\
(1-2-3)\end{array}$ & $\begin{array}{l}\text { p-value } \\
(1-2-3)\end{array}$ & $\begin{array}{l}\text { Statistics } \\
(1-2)\end{array}$ & $\begin{array}{l}P \text {-value } \\
(I-2)\end{array}$ \\
\hline \multicolumn{8}{|l|}{ VAS measurements, $0-10$, median(IQR) } \\
\hline Prebiopsy procedure & $6.0(3.0)$ & $6.0(4.0)$ & $6.0(4.0)$ & 1.074 & 0.584 & & \\
\hline Intrabiopsy procedure & $3.0(2.0)$ & $4.0(3.0)$ & $6.0(3.0)$ & 29.345 & $<0.001$ & 581.00 & 0.048 \\
\hline Change from pre- to Intrabiopsy & $2.0(1.0)$ & $2.0(0.0)$ & $0.0(5.0)$ & 20.230 & $<0.001$ & 586.00 & 0.036 \\
\hline \multicolumn{8}{|l|}{ SATI measurements, 0-I0, median(IQR) } \\
\hline Prebiopsy procedure & $18.0(6.0)$ & $19.0(7.0)$ & $18.0(7.0)$ & 2.145 & 0.342 & & \\
\hline Postbiopsy procedure & $8.0(3.0)$ & $10.0(5.0)$ & $14.0(5.0)$ & 57.663 & $<0.001$ & 371.50 & $<0.001$ \\
\hline Change from pre- to postbiopsy & $10.0(3.0)$ & $8.0(2.0)$ & $4.0(2.0)$ & 74.937 & $<0.001$ & 399.50 & $<0.001$ \\
\hline \multicolumn{8}{|l|}{ Other postprocedure variables } \\
\hline Satisfaction, 0-10, median(IQR) & $8.0(3.0)$ & $8.0(4.0)$ & $6.0(4.0)$ & $14.17 \mid$ & 0.001 & 0.553 & 0.574 \\
\hline Willingness to repeat biopsy $\mathrm{n}(\%)$ & $38(95.0)$ & $36(92.3)$ & $31(75.6)$ & 8.181 & 0.017 & $0.24 I$ & 0.623 \\
\hline
\end{tabular}

Note: Significant $p$-values are presented in bold.

Abbreviations: HR, hand holding with relatives; HS, hand holding with strangers; VAS, visual analog scale; IQR, interquartile range; SATI, state-trait anxiety inventory.

However, there was no significant difference between the HR group and the HS group $(P=0.148)$.

After TRUS-PBx, participants' anxiety levels were significantly lower in the hand-holding groups $(P<0.001)$ than in the control group. After the procedure, anxiety levels were significantly lower in the HR group than in the HS group $(P=0.019)$.

The satisfaction of the patients in the HR group and HS group was significantly higher than that of those in the control group ( $P=0.001)$, and they were more willing to undergo repeated TRUS-PBx $(P=0.017)$.

The hemodynamic changes in the three groups are shown in Figure 1. The change in SBP before and after TRUS-PBx in the intervention group was significantly less than that in the control group $(P=0.006)$, but there was no significant difference in DBP $(P=0.622)$. There was no significant difference in the change in SBP between the HR group and the HS group $(P=0.442)$.

The heart rate fluctuation during TRUS-PBx in the intervention group was significantly less than that in the control group $(P<0.001)$. Furthermore, the heart rate in the HR group changed less than that in the HS group $\left(P_{\text {pre-intra }}=0.008, P_{\text {intra- }}\right.$ post $=0.001$ ).

\section{Discussion}

$\mathrm{PCa}$ is a common disease and the second most common cancer in men worldwide. With the increase in life expectancy, the westernization of diet structure and the continuous improvement of diagnostic technology in China, an increasing number of PCa patients have been diagnosed in recent years. ${ }^{12}$ TRUS-PBx is an essential diagnostic modality for PCa patients with elevated PSA or abnormal DRE or prostate imaging.

TRUS-PBx is well tolerated in most patients, but 19$25 \%$ of patients suffer from moderate or severe pain. ${ }^{3}$ Bastide et al reported that $80 \%$ of patients who did not receive local anesthesia experienced significant discomfort, and approximately half of patients received less than 6 core biopsies. ${ }^{13}$ To improve the positive rate of $\mathrm{PBx}$, many physicians have gradually increased the number of biopsy needles. However, patients who underwent more core biopsies felt more severe pain, and some patients could not successfully complete PBx. Sometimes, they refused to undergo re-PBx, even against doctors' advice, due to pain.

Through MRI, it can be predicted which patients will feel more pain. These findings implicate that (i) biopsynaive patients, (ii) patients with a larger prostate, (iii) patients with a shorter prostate-anus surface distance, and (iv) patients with a narrow anorectal angle are likely to experience more severe pain during biopsy. ${ }^{14}$ Accordingly, few studies have indicated that when effective analgesia is administered, the number of biopsy cores taken does not increase the level of pain. ${ }^{15}$ Methods currently employed for pain control during prostate biopsy include intrarectal local anesthetic (IRLA), periprostatic nerve block (PPNB), caudal block, sedation anesthesia, and spinal anesthesia. Prilocaine is similar to lidocaine in terms of speed of onset but provides longer-term pain control. ${ }^{16}$ 


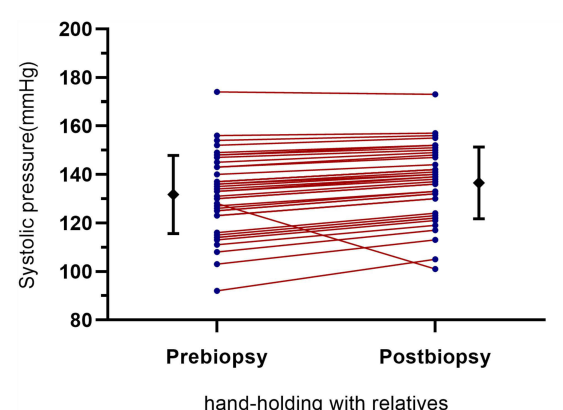

hand-holding with relatives
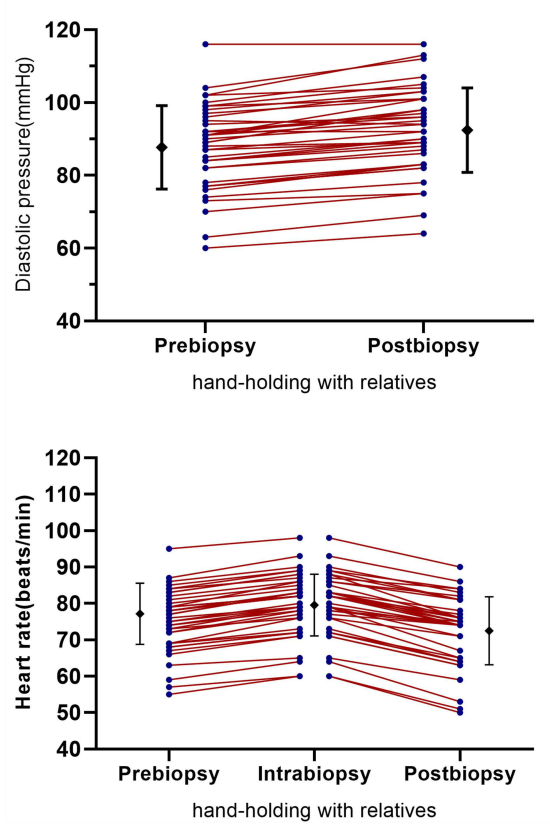

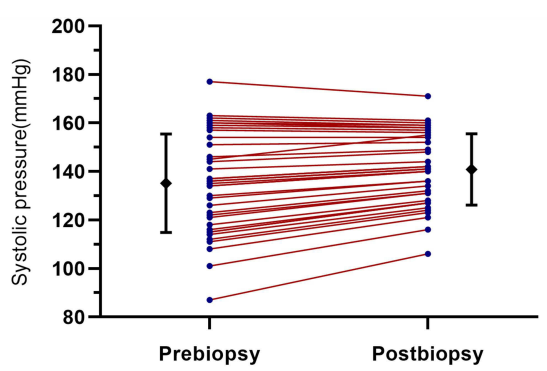

hand-holding with strangers
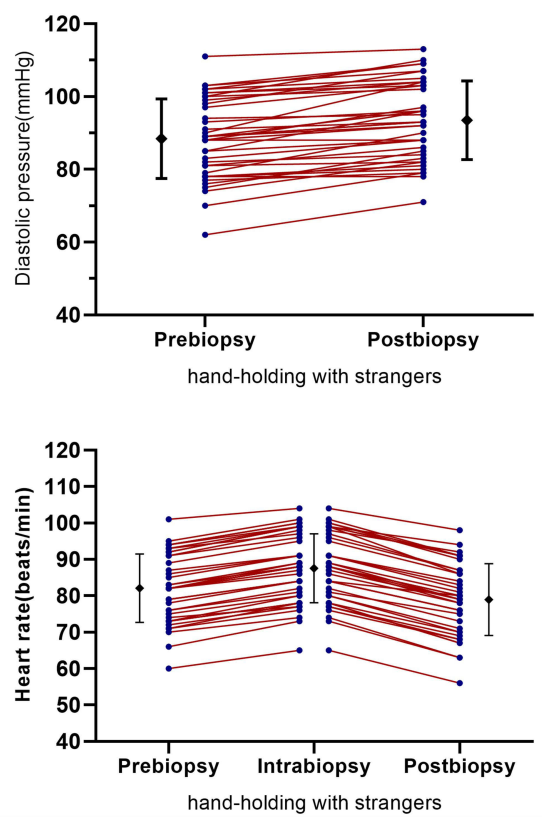
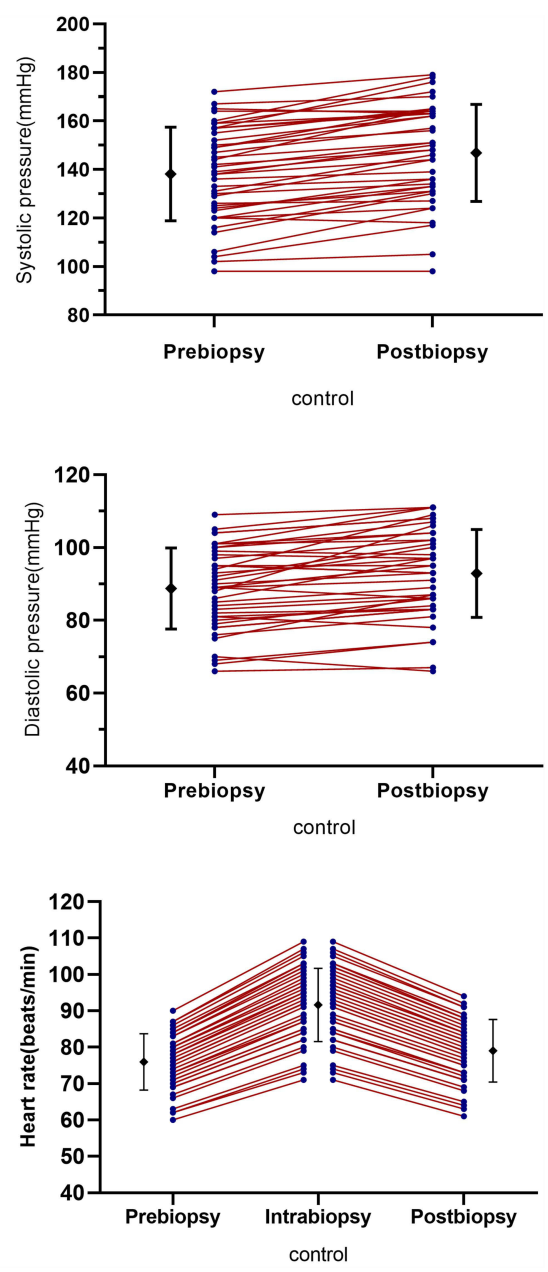

Figure I Comparison of hemodynamic parameters measured during PBx among the three groups.

Despite the use of effective anesthesia and lubrication, patients may still experience pain during TRUS-PBx, which may be related to their level of anxiety during the procedure. ${ }^{17}$ Many authors have shown that the negative impact of PBx on patient well-being can even begin while the patient is waiting to undergo the procedure and that patients experience increased anxiety and worry immediately before undergoing biopsy, with a minority (20\%) suffering from high levels of distress at that time. The use of complementary therapies to decrease pain and anxiety during standard endoscopic procedures is becoming increasingly widespread. These therapies include music, transcutaneous electrical nerve stimulation, acupuncture, and auricular acupressure. The least invasive of these therapies is music. ${ }^{5,18}$

This study found that patients who received gentle hand holding, especially from relatives, felt more supported and encouraged. When researchers asked participants how they felt during a prostate biopsy, patients in the
HR or HS group evaluated their experience positively. They felt comforted when someone was near their bed and holding their hands. In fact, the STAI score, pain VAS score, satisfaction score, and willingness to perform more prostate biopsies were significantly better in the hand-holding group than in the control group $(P<0.05)$. This shows that hand holding can reduce patient anxiety and pain, which leads to a better medical experience and the possibility of repeated biopsy.

The hand-holding analgesic effect can be explained by the well-known distraction effect. ${ }^{19,20}$ However, we found that when patients held hands with family members, the pain and anxiety they suffered were relieved more than when they held hands with strangers $(P<0.05)$, so there may be other explanations for this phenomenon. Recent experimental studies have shown that a particular type of dynamic touch may be a particularly effective and salient embodied form of communicating active social support. $^{21}$ 
The touch of the observer enhances the coupling, which increases the tendency of the target to be understood, which in turn activates the reward mechanism. ${ }^{22}$ Consistent with this, recent research has shown that the reward circuit is activated when humans experience synchronization. $^{23}$ Therefore, brain-to-brain coupling between the observer and the target experiencing pain may be influenced by social understanding, which may be beneficial or lead to analgesia. In addition, social touch can convey empathy between the target and the observer, and social understanding can affect pain management by increasing rewards. Research supporting this idea suggests that partner touch can enhance pain relief, and trait empathy can predict the extent of relief. ${ }^{24}$

In addition, researchers have demonstrated that from the perspective of self and others, physiological activation patterns in painful and pleasant virtual reality scenarios are similar, and there are shared brain activation patterns in pleasant and unpleasant touches. ${ }^{25,26}$ Therefore, interpersonal touch, especially between spouses, can increase empathy sharing, help the observer feel the target's pain, and give emotional support to the pain target, resulting in pain relief and manifesting as interpersonal physiological coupling. ${ }^{27,28}$

The STAI and VAS tools used to quantify anxiety and pain are subjective. Therefore, as indirect indicators of anxiety and pain, hemodynamic measurements were taken pre-, intra- and postprocedure. In this study, the SBP and heart rate fluctuations in the HR and HS groups were smaller than those in the control group $(P<0.01)$, but DBP did not change significantly in any group $(P=0.622)$. The results were similar to previous studies in other fields, such as cataract surgery and percutaneous vertebroplasty, in which hand holding by relatives or nurses reduced anxiety and pain and smoothed fluctuations in SBP but not DBP. ${ }^{10}$

Our study also found that the change in heart rate in the HR group was significantly smaller than that in the HS group $(P<0.01)$. This finding could be explained by the fact that the quality of a relationship regulates the analgesic effects of social support. In fact, most studies show social support through close spousal relationships, ${ }^{7,8}$ whereas the participants in our study were supported not only by spouses but also by family members, which reflects the extensive level of social support in daily life. These types of relationships may not have the same effect as a romantic partner, but they can still be effective in alleviating the threat of pain and anxiety.
There are several limitations in this study. Fusion biopsy was not performed, at least in secondary patients. We did not assess the pain that patients felt at each step of the procedure, such as during injection of oxybuprocaine gel, insertion of the TRUS probes into the rectum and penetration of the biopsy needle into the prostate tissue. Moreover, it is impossible to double-blind patients or doctors, so patients are susceptible to the Hawthorne effect. As a result, the data interpretation or reporting of patient anxiety and pain may be biased.

\section{Conclusion}

Despite these limitations, the results of this study suggest that hand holding, especially with relatives, is very effective in reducing anxiety and pain during prostate biopsy. Hand holding is a noninvasive intervention that can be performed without the use of special equipment. Patient cooperation is key to the completion of prostate biopsy, and hand holding can comfort the patient during PBx. Hence, we recommend hand holding with relatives as an effective adjunct during PBx.

\section{Data Sharing Statement}

The datasets used and/or analyzed during the current study are available from the corresponding author on reasonable request.

\section{Ethics Approval and Consent to Participate}

This study was conducted according to the ethical principles of the Declaration of Helsinki. Approval was granted by the Ethics Committee of the Ninth People's Hospital, Shanghai Jiao Tong University School of Medicine (No. SH9H-2020-T40-1) and registered with clinicaltrials.gov (ChiCTR2000032238). All study participants provided written informed consent before inclusion in this study. Patient data were handled according to the applicable laws and regulations. A document that linked the study codes to the patients' identifying information was digitally stored and protected.

\section{Funding}

We are thankful for the financial support of the Science Market of the Shanghai Science and Technology Committee (19dz2320600). 


\section{Disclosure}

Wenfeng Li and Yuanshen Mao should be considered cofirst authors. The authors declare that they have no competing interests.

\section{References}

1. Kucur M, Goktas S, Kaynar M, et al. Selective low-dose spinal anesthesia for transrectal prostate biopsy: a prospective and randomized study. J Endourol. 2015;29(12):1412-1417. doi:10.1089/ end.2015.0450

2. Packiam VT, Nottingham CU, Cohen AJ, Eggener SE, Gerber GS No effect of music on anxiety and pain during transrectal prostate biopsies: a randomized trial. Urology. 2018;117:31-35. doi:10.1016/j. urology.2018.04.014

3. Zisman A, Leibovici D, Kleinmann J, Siegel YI, Lindner A. The impact of prostate biopsy on patient well-being: a prospective study of pain, anxiety and erectile dysfunction. J Urol. 2001;165 (2):445-454. doi:10.1097/00005392-200102000-00023

4. Li M, Wang $\mathrm{Z}$, $\mathrm{Li} \mathrm{H}$, et al. Local anesthesia for transrectal ultrasound-guided biopsy of the prostate: a meta-analysis. Sci Rep. 2017;7:1-8. doi:10.1038/srep40421

5. Kim DK, Lee JY, Jung JH, et al. What is the most effective local anesthesia for transrectal ultrasonography-guided biopsy of the prostate? A systematic review and network meta-analysis of 47 randomized clinical trials. Sci Rep. 2019;9(1):1-14. doi:10.1038/s41598-019-41412-w

6. Dell'Atti L. Impact of music on anxiety and pain perception among men undergoing prostate biopsy: synthesis of qualitative literature. Complement Ther Clin Pract. 2021;43:101330. doi:10.1016/j. ctcp. 2021.101330

7. Che X, Cash R, Fitzgerald P, Fitzgibbon BM. The social regulation of pain: autonomic and neurophysiological changes associated with perceived threat. J Pain. 2018;19(5):496-505. doi:10.1016/j.jpain.2017.12.007

8. Graff TC, Luke SG, Birmingham WC. Supportive hand-holding attenuates pupillary responses to stress in adult couples. PLoS One. 2019;14(2):1-20. doi:10.1371/journal.pone.0212703

9. Coan JA, Schaefer HS, Davidson RJ. Lending a hand: social regulation of the neural response to threat. Psychol Sci. 2006;17 (12):1032-1039. doi:10.1111/j.1467-9280.2006.01832.x

10. Kim BH, Kang HY, Choi EY. Effects of handholding and providing information on anxiety in patients undergoing percutaneous vertebroplasty. J Clin Nurs. 2015;24(23-24):3459-3468. doi:10.1111/ jocn. 12928

11. Bayrampour H, McDonald S, Fung T, Tough S. Reliability and validity of three shortened versions of the State Anxiety Inventory scale during the perinatal period. J Psychosom Obstet Gynaecol. 2014;35(3):101-107. doi:10.3109/0167482X.2014.950218

12. Roberts MH, Klatzkin RR, Mechlin B. Social support attenuates physiological stress responses and experimental pain sensitivity to cold pressor pain. Ann Behav Med. 2015;49(4):557-569. doi:10.1007/s12160-015-9686-3

13. Bastide C, Lechevallier E, Eghazarian C, Ortega JC, Coulange C. Tolerance of pain during transrectal ultrasound-guided biopsy of the prostate: risk factors. Prostate Cancer Prostatic Dis. 2003;6 (3):239-241. doi:10.1038/sj.pcan.4500664
14. Sonmez G, Tombul ST, Demirtas T, Demirtas A. Risk factors associated with pain in fusion prostate biopsy. Prostate Int. 2020;8 (4):185-189. doi:10.1016/j.prnil.2020.05.004

15. Demirtaş A, Sönmez G, Tombul ŞT, Demirtaş T. Comparison of pain levels in fusion prostate biopsy and standard TRUS-guided biopsy. Int Braz $j$ Urol. 2020;46(4):557-562. doi:10.1590/s1677-5538. ibju.2019.0154

16. Sönmez G, Baydilli N, Tombul ŞT, Güler G, Demirtaş A. Comparison of lidocaine and prilocaine efficiencies in periprostatic nerve block for transrectal prostate biopsy: a randomized prospective study. Bull Urooncol. 2019;18(1):1-5. doi:10.4274/uob.galenos.20 18.1096

17. Grinberg AS, Sellinger JJ, Sprenkle PC, et al. Effect of diaphragmatic breathing on procedural anxiety during transrectal prostate biopsy. Urology. 2020;137:26-32. doi:10.1016/j.urology.2019.12.024

18. Cho SW, Choi HJ. Effect of music on reducing anxiety for patients undergoing transrectal ultrasound-guided prostate biopsies: randomized prospective trial. Urol J. 2016;13(2):2612-2614. doi:10.22 037/uj.v13i2.3187

19. Kwon WA, Lee JW, Seo HK, et al. Hand-holding during cystoscopy decreases patient anxiety, pain, and dissatisfaction: a pilot randomized controlled trial. Urol Int. 2018;100(2):222-227. doi:10.1159/ 000485745

20. Gezginci E, Iyigun E, Kibar Y, Bedir S. Three distraction methods for pain reduction during cystoscopy: a randomized controlled trial evaluating the effects on pain, anxiety, and satisfaction. $J$ Endourol. 2018;32(11):1078-1084. doi:10.1089/end.2018.0491

21. Bernardes SF, Forgeron P, Fournier K, Reszel J. Beyond solicitousness: a comprehensive review on informal pain-related social support. Pain. 2017;158(11):2066-2076. doi:10.1097/j.pain.000000 0000001033

22. Morelli SA, Torre JB, Eisenberger NI. The neural bases of feeling understood and not understood. Soc Cogn Affect Neurosci. 2014;9 (12):1890-1896. doi:10.1093/scan/nst191

23. Chatel-Goldman J, Congedo M, Jutten C, Schwartz JL. Touch increases autonomic coupling between romantic partners. Front Behav Neurosci. 2014;8:95. doi:10.3389/fnbeh.2014.00095

24. Goldstein P, Shamay-Tsoory SG, Yellinek S, Weissman-Fogel I. Empathy predicts an experimental pain reduction during touch. J Pain. 2016;17(10):1049-1057. doi:10.1016/j.jpain.2016.06.007

25. Lamm C, Silani G, Singer T. Distinct neural networks underlying empathy for pleasant and unpleasant touch. Cortex. 2015;70:79-89. doi:10.1016/j.cortex.2015.01.021

26. Fusaro M, Tieri G, Aglioti SM. Seeing pain and pleasure on self and others: behavioral and psychophysiological reactivity in immersive virtual reality. J Neurophysiol. 2016;116(6):2656-2662. doi:10.1152/ jn.00489.2016

27. von Mohr M, Krahé C, Beck B, Fotopoulou A. The social buffering of pain by affective touch: a laser-evoked potential study in romantic couples. Soc Cogn Affect Neurosci. 2018;13(11):1121-1130. doi:10.1093/scan/nsy085

28. Goldstein P, Weissman-Fogel I, Dumas G, Shamay-Tsoory SG. Brain-to-brain coupling during handholding is associated with pain reduction. Proc Natl Acad Sci U S A. 2018;115(11):E2528-E2537. doi:10.1073/pnas.1703643115 


\section{Publish your work in this journal}

Patient Preference and Adherence is an international, peer-reviewed, open access journal that focusing on the growing importance of patient preference and adherence throughout the therapeutic continuum. Patient satisfaction, acceptability, quality of life, compliance, persistence and their role in developing new therapeutic modalities and compounds to optimize clinical outcomes for existing disease

Submit your manuscript here: https://www.dovepress.com/patient-preference-and-adherence-journa states are major areas of interest for the journal. This journal has been accepted for indexing on PubMed Central. The manuscript management system is completely online and includes a very quick and fair peer-review system, which is all easy to use. Visit http:// www.dovepress.com/testimonials.php to read real quotes from published authors. 